Sharif University of Technology
Scientia Iranica
SCIENTIA
IRAN Transactions B: Mechanical Engineering
www.scientiairanica.com

\title{
Aeroelastic analysis of a typical section using Euler and Navier-Stokes mesh-less method
}

\author{
S. Sattarzadeh, A. Jahangirian* and H. Shahverdi \\ Department of Aerospace Engineering, Amirkabir University of Technology, 424 Hafez Avenue, Tehran, P.O. Box 15875-4413, Iran.
}

Received 23 April 2014; received in revised form 2 August 2014; accepted 13 April 2015

\author{
KEYWORDS \\ Aeroelastic instability; \\ Compressible flow; \\ Flutter speed; \\ Mesh-less method; \\ Navier-Stokes \\ equations; \\ Transonic flow.
}

\begin{abstract}
The main aim of this paper is to develop an efficient aeroelastic tool for predicting the flutter speed of a typical section in transonic regime. An implicit meshless method, based on Euler and Navier-Stokes equations, is conducted to simulate the transonic fluid flow around an airfoil. This technique is applied directly to the differential form of the aerodynamic governing equations and the time integration is carried out using a dual-time implicit time discretization scheme. The capabilities of the flow solution method are demonstrated by flow computations around NACA0012 airfoil under different flow conditions. For structural dynamics simulation, a typical section model with pitching and plunging motion capability is considered. Finally, the aeroelastic analysis of the 2D model is performed by the consecutive simulation of both structural and aerodynamic domains. Also, the effect of viscosity and time interval choice between two structural and aerodynamic solvers on flutter instability is studied. A comparison between the obtained results and those available in the literature shows the good accuracy of the present method.

(C) 2016 Sharif University of Technology. All rights reserved.
\end{abstract}

\section{Introduction}

There is no escaping the fact that aeroelastic stability investigations play an important role in the designing process of an air vehicle. The most performed activities and research in the aeroelastic field have been determination of critical flutter speed. In this regard, finding a suitable and powerful method for solving structural and fluid flow fields has always been an attractive subject for researchers [1,2]. Specifically, from a fluid computational aspect, some well-known analytical models, such as Theodorsen's theory for unsteady subsonic incompressible flow and the piston theory for supersonic applications, are reachable (of course with some limitations) [3]. Moreover, the Computational Fluid Dynamics (CFD) method can be

*. Corresponding author. Tel.: +982164543223

E-mail addresses: sattarzadeh@aut.ac.ir (S. Sattarzadeh); ajahan@aut.ac.ir(A.Jahangirian); h_shahverdi@aut.ac.ir (H. Shahverdi) put into use to study the complex phenomena of a fluid flow (e.g. flow separation, compressibility, shock, etc.). Using these methods may increase solution complexity and computational effort, and, thus, solution of an especial case, such as a transonic flow regime, with a suitable tool, is an important challenge in aeroelastic analysis. Transonic aeroelasticity is more complex in comparison with subsonic and supersonic regimes due to the existence of shock waves across the airfoil. In these regimes, the fluid flow equations can be used in the linear form, which could be incorporated into aeroelastic equations. However, transonic flow has a nonlinear nature that is not easy to be solved with the same techniques. One way to overcome these difficulties is to use numerical methods (CFD) which may be implemented through time-marching schemes.

Finite element or finite volume methods are extensively applied to address the computational aeroelasticity field problems [4-6]. However, most of these studies have some restrictions in transonic flow. The first aeroelastic study in the transonic regime was 
performed by Edwards et al. [2]. In this paper, a nonlinear time-marching aeroelastic model was solved using Transonic Small Disturbance (TSD). Bendiksen and Kousen achieved the flutter boundaries for a NACA 64A010 airfoil using an explicit method based on the convolution integral. They found that the largeamplitude limit cycles could be achieved in unsteady motion [7]. Lee studied the effect of viscosity using the Finite Volume (FV) method [8]. Kholodar et al. [9] applied a novel Harmonic Balance (HB) technique to solve the flutter boundary in the presence of Euler equations. In other work by Thomas et al. [10,11], this method was developed for $\mathrm{N}-\mathrm{S}$ equations to predict flutter velocity. The effect of viscosity on flutter velocity in the transonic regime, based on the $\mathrm{HB}$ method, was investigated by Schwarz et al. [12].

Guruswamy [13] attained acceptable results using different equations for 2D and 3D geometries, including vertical flow. To capture valid results in these methods, a refined mesh is required. Indeed, the problem of these methods will be initiated when the complexity of the model and its operational conditions are increased.

Compared to mesh-based methods, mesh-less methods have some advantages. For example, the main problem of Computational Fluid Dynamics (CFD) in mesh-based methods is their difficulties in generation of a practical mesh [14]. But, in mesh-less methods, only computational points (instead of elements) are used in the solution process. One of the major disadvantages of all mesh-based methods (especially in aero-elastic analysis) is their difficulty in the solution of unsteady flow because of element deformation. This obstacle could be resolved using mesh-less methods, which employ computational points that are easily replaced and moved in comparison with mesh-based algorithms. This privilege can be useful especially in unsteady conditions (because of a large number of time steps in unsteady computations). These advantages encourage the use of mesh-less method in aero-elastic applications. Different mesh-less methods have been presented in the literature $[15,16]$. A very efficient implicit mesh-less method is applied to solve steady compressible flows by Jahangirian and Hashemi [17]. In that paper, the least square method, based on the Taylor series, was applied to calculate the derivatives. The results indicated that the computational time is decreased by $50 \%$ in comparison with the similar Control Volume (CV) method using the same point distribution [18]. In this paper, implicit and explicit methods were developed to solve unsteady stationary flows. The unsteady mesh-less method, based on point replacement, has been provided by Wang et al. [19] and Ortega et al. [20]. In another work, Wang et al. [21] used Delaunay triangle principles to solve unsteady flow.

Several mesh-less methods have been applied for fluid-solid interaction problems [22-25]. For instance, $\mathrm{Hu}$ et al. [22] applied the Pure Particle Method (PPM) to complex geometries, along with large deformation capability. In another work, a staggered algorithm, based on the mesh-less method, has been extended for fluid-structural interaction [23]. However, only a few works have been performed based on the least square method.

The main objective of the present work is to further extend the application of the least square meshless method to aeroelastic moving boundary unsteady problems under transonic flow conditions. At the first stage, the ability of the flow solution method is demonstrated. It is shown that the convergence rate of this method is higher than the similar Control Volume (CV) method with the same discretization and initial data [18]. In the next step, the ability of the method is shown regarding the solution of unsteady flow. Then, the provided computational aerodynamic model is incorporated into the system of aeroelastic equations of a typical section model to perform aeroelastic analysis. Also, the effect of viscosity on flutter instability is studied.

\section{Computational models}

\subsection{Aerodynamic model}

The fluid flow around a moving, two-dimensional airfoil is governed by Navier-Stokes (N-S) equations, which can be written in the differential form as [17]:

$$
\begin{aligned}
{\left[\frac{\partial \mathbf{w}}{\partial t}+\mathbf{w} \nabla \bullet \mathbf{w}_{s}\right] } & +\left[\frac{\partial \mathbf{f}^{I}}{\partial x}+\frac{\partial \mathbf{g}^{I}}{\partial y}\right] \\
& =\frac{\operatorname{Ma}_{\infty}}{\operatorname{Re}_{\infty}}\left[\frac{\partial \mathbf{f}^{V}}{\partial x}+\frac{\partial \mathbf{g}^{V}}{\partial y}\right],
\end{aligned}
$$

where:

$$
\begin{aligned}
& \mathbf{w}=\left(\begin{array}{c}
\rho \\
\rho u \\
\rho v \\
\rho E
\end{array}\right), \quad \mathbf{w}_{s}=\left(\begin{array}{l}
x_{t} \\
y_{t}
\end{array}\right) \quad \mathbf{f}^{I}=\left(\begin{array}{c}
\rho U \\
\rho u U+P \\
\rho v U \\
\rho E U+P u
\end{array}\right), \\
& \mathbf{g}^{l}=\left(\begin{array}{c}
\rho V \\
\rho u V \\
\rho v V+P \\
\rho E V+P v
\end{array}\right), \quad \mathbf{f}^{V}=\left(\begin{array}{c}
0 \\
\tau_{x x} \\
\tau_{x y} \\
u \tau_{x x}+v \tau_{x y}-q_{x}
\end{array}\right), \\
& \mathbf{g}^{V}=\left(\begin{array}{c}
0 \\
\tau_{x y} \\
\tau_{y y} \\
u \tau_{x y}+v \tau_{y y}-q_{y}
\end{array}\right) .
\end{aligned}
$$

$U$ and $V$ represent the $x$ and $y$ components of relative velocity and are evaluated as:

$$
U=x-x_{t}, \quad V=v-y_{t} .
$$




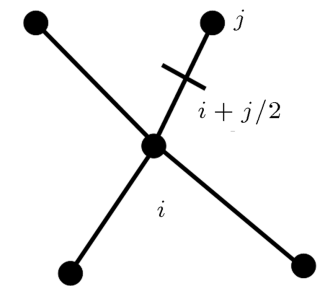

Figure 1. A sample point and its neighbors.

For a perfect gas, the following equation can be written as:

$$
P=(\gamma-1)\left[\rho E-\frac{\rho\left(u^{2}+v^{2}\right)}{2}\right] .
$$

In this study, for applying the mesh-less method, equations are used in a conservation form. In this method, the differential form of governing equations is implemented, and a least-square approximation is used to calculate the derivatives [26]. According to Figure 1, $C_{i}$ is the set of computational points, which are neighbors for point $i$, and the value of any parameter, $\phi$, is defined at the mid-point between two adjacent points [27]. The amount of function $\phi_{i j}$ is assumed to change linearly along line $i j$. Using Taylor's formula for point $i$ and its neighboring points, the following equation is achieved [17]:

$$
\begin{aligned}
& \left(\frac{\partial \phi}{\partial x}\right) \Delta x_{i j}+\left(\frac{\partial \phi}{\partial y}\right) \Delta y_{i j}=\Delta \phi_{i j}, \Delta x_{i j}=x_{j}-x_{i} \\
& \Delta y_{i j}=y_{j}-y_{i}, \quad \Delta \phi_{i j}=\phi_{j}-\phi_{i} .
\end{aligned}
$$

Similar equations are achieved for all cloud points which are neighbors with point $i$ by considering an arbitrary weighting factor, $\omega_{i}$. This may result in the following matrix for point $i[26]$ :

$$
\begin{aligned}
& \left(\begin{array}{cc}
\omega_{i 1} \Delta x_{i 1} & \omega_{i 1} \Delta y_{i 1} \\
\cdots & \cdots \\
\omega_{i m} \Delta x_{i m} & \omega_{i m} \Delta y_{i m}
\end{array}\right)\left[\begin{array}{c}
\left.\frac{\partial \phi}{\partial x}\right|_{i} \\
\left.\frac{\partial \phi}{\partial y}\right|_{i}
\end{array}\right]=\left[\begin{array}{c}
\omega_{i 1} \Delta \phi_{i 1} \\
\cdots \\
\omega_{i m} \Delta \phi_{i m}
\end{array}\right], \\
& \omega_{i j}=\frac{1}{d_{i j}} .
\end{aligned}
$$

By considering Eq. (5) and using the least-squares method, the derivatives of each parameter can be estimated as follows [26]:

$$
\left.\frac{\partial \phi}{\partial x}\right|_{i}=\sum_{j=1}^{m} a_{i j} \Delta \phi_{i j},\left.\quad \frac{\partial \phi}{\partial y}\right|_{i}=\sum_{j=1}^{m} b_{i j} \Delta \phi_{i j} .
$$

The coefficients in Eq. (7) can be computed through solving Eq. (6) [16]:

$$
\begin{aligned}
& a_{i j}= \\
& \frac{\omega_{i j} \Delta x_{i j} \sum_{k=1}^{m} \omega_{i k} \Delta y_{i k}^{2}-\omega_{i j} \Delta y_{i j} \sum_{k=1}^{m} \omega_{i k} \Delta x_{i k} \Delta y_{i k}}{\sum_{k=1}^{m} \omega_{i k} \Delta x_{i j}^{2} \sum_{k=1}^{m} \omega_{i k} \Delta y_{i k}^{2}-\left(\sum_{k=1}^{m} \omega_{i k} \Delta x_{i k} \Delta y_{i k}\right)^{2}}
\end{aligned}
$$

$$
\begin{aligned}
& b_{i j}= \\
& \frac{\omega_{i j} \Delta y_{i j} \sum_{k=1}^{m} \omega_{i k} \Delta x_{i k}^{2}-\omega_{i j} \Delta x_{i j} \sum_{k=1}^{m} \omega_{i k} \Delta x_{i k} \Delta y_{i k}}{\sum_{k=1}^{m} \omega_{i k} \Delta x_{i j}^{2} \sum_{k=1}^{m} \omega_{i k} \Delta y_{i k}^{2}-\left(\sum_{k=1}^{m} \omega_{i k} \Delta x_{i k} \Delta y_{i k}\right)^{2}} .
\end{aligned}
$$

To achieve a semi-discrete form of the Navier-Stokes equations (Eq. (1)) at point $i$, using Eq. (7), the following equation is obtained [16]:

$$
\begin{gathered}
{\left[\frac{\partial \mathbf{w}_{i}}{\partial t}+\mathbf{w}_{i}\left(\sum_{j=1}^{m} a_{i j} \Delta x_{t, i j}+\sum_{j=1}^{m} b_{i j} \Delta y_{t, i j}\right)\right]} \\
+\left[\sum_{j=1}^{m} a_{i j} \Delta \mathbf{f}_{i j}^{I}+\sum_{j=1}^{m} b_{i j} \Delta \mathbf{g}_{i j}^{I}\right] \\
=\frac{\operatorname{Ma}_{\infty}}{\operatorname{Re}_{\infty}}\left[\sum_{j=1}^{m} a_{i j} \Delta \mathbf{f}_{i j}^{V}+\sum_{j=1}^{m} b_{i j} \Delta \mathbf{g}_{i j}^{V}\right] \\
i=1 \ldots N .
\end{gathered}
$$

In this equation, $\Delta \mathbf{f}_{i j}$ and $\Delta g_{i j}$ are:

$$
\Delta \mathbf{f}_{i j}=\mathbf{f}_{j}-\mathbf{f}_{i}, \quad \Delta \mathbf{g}_{i j}=\mathbf{g}_{j}-\mathbf{g}_{i} .
$$

By defining $H=a F+b G$ (which is defined as flux in the direction of the least square coefficients and is similar to flux which is calculated in the mesh-based methods [17]) in Eq. (9), the following equation can be achieved:

$$
\begin{aligned}
\left(\frac{\partial \mathbf{w}_{i}}{\partial t}\right. & \left.+\mathbf{w}_{i}\left(\sum_{j=1}^{m} a_{i j} \Delta x_{t, i j}+\sum_{j=1}^{m} b_{i j} \Delta y_{t, i j}\right)\right) \\
& +\sum_{j=1}^{m} \Delta \mathbf{H}_{i j}^{I}=\frac{\mathrm{Ma}_{\infty}}{\operatorname{Re}_{\infty}}\left[\sum_{j=1}^{m} \Delta \mathbf{H}_{i j}^{V}\right] \\
\Delta \mathbf{H}_{i j} & =\mathbf{H}_{j}-\mathbf{H}_{i} .
\end{aligned}
$$

By applying the central difference method to the Navier-Stokes equations, the following equation can be achieved:

$$
\begin{aligned}
\left(\frac{\partial \mathbf{w}_{i}}{\partial t}\right. & \left.+\mathbf{w}_{i}\left(\sum_{j=1}^{m} a_{i j} \Delta x_{t, i j}+\sum_{j=1}^{m} b_{i j} \Delta y_{t, i j}\right)\right) \\
& +2 \sum_{j=1}^{m} \Delta \mathbf{H}_{i j+1 / 2}^{I}=\frac{2 \mathrm{Ma}_{\infty}}{\operatorname{Re}_{\infty}}\left[\sum_{j=1}^{m} \Delta \mathbf{H}_{i j+1 / 2}^{V}\right] \\
\mathbf{H}_{j+1 / 2} & =\frac{\mathbf{H}_{i}+\mathbf{H}_{j}}{2} .
\end{aligned}
$$

Due to the use of the central difference method, 
unstable results are achieved. In order to overcome this problem, stabilizing terms are used in Eq. (9) by adding damping terms. In this dissipation model, in order to prevent oscillations, especially in critical zones, an aggregation of the second and fourth differences of conserved variables $(W)$ is added to Eq. (11) [17], which is denoted by the $D$ symbol in the following relation:

$$
\begin{aligned}
\frac{\partial \mathbf{w}_{i}}{\partial t} & +2\left(\sum_{j=1}^{m} \Delta \mathbf{H}_{i j+1 / 2}^{I}-\frac{\mathrm{Ma}_{\infty}}{\operatorname{Re}_{\infty}}\left[\sum_{j=1}^{m} \Delta \mathbf{H}_{i j+1 / 2}^{V}\right]\right) \\
& -\mathbf{D}_{i}=0 .
\end{aligned}
$$

These dissipation terms are defined by:

$$
\begin{aligned}
& D_{i}=\left(\nabla\left(\varepsilon^{(2)} \lambda\right) \nabla W-\nabla^{2}\left(\varepsilon^{(4)} \lambda\right) \nabla^{2} W\right)_{i}, \\
& \nabla\left(\varepsilon^{(2)} \lambda\right) \nabla W=\sum_{j=1}^{n}\left[\left(\varepsilon^{(2)} \lambda\right)_{i, j / 2}\left(W_{j}-W_{i}\right)\right], \\
& \nabla^{2} W=\sum_{j=1}^{n}\left(W_{j}-W_{i}\right),
\end{aligned}
$$

where $\varepsilon^{(2)}$ and $\varepsilon^{(4)}$ can be formulated as:

$$
\begin{aligned}
\varepsilon_{i j}^{(2)} & =k_{2} v_{i j}, \\
\varepsilon_{i j}^{4} & =\max \left(0, k_{4}-\varepsilon_{i j}^{(2)}\right), \\
v_{i j} & =\frac{\left|P_{j}-P_{i}\right|}{\left|P_{j}+P_{i}\right|} .
\end{aligned}
$$

The values of constant $k_{2}$ and $k_{4}$ are in the range $0<k_{2}<1$ and $\frac{1}{256}<k_{4}<\frac{1}{32}$ [28]. Eq. (11) is applied to each node in the computational domain and a set of ordinary differential equations are obtained as follows [28]:

$$
\begin{aligned}
& \frac{\partial W_{i}}{\partial t}+R\left(W_{i}\right)=0, \\
& R_{i}(W)=W_{i}\left(\sum_{j=1}^{m} a_{i j} \Delta x_{t, i j}+\sum_{j=1}^{m} b_{i j} \Delta y_{t, i j}\right) \\
& \quad+\sum_{j=1}^{m} \Delta H_{i j}^{I}-\frac{\operatorname{Ma}_{\infty}}{\operatorname{Re}_{\infty}}\left[\sum_{j=1}^{m} \Delta H_{i j}^{V}\right]-D_{i}, \\
& \frac{d W}{d t}+R\left(W_{i}^{n+1}\right)=0 .
\end{aligned}
$$

An implicit time discretization is applied in Eq. (16), which can be written as [17]:

$$
\frac{\partial \mathbf{w}_{i}^{n+1}}{\partial t}+\mathbf{R}_{i}\left(\mathbf{w}^{n+1}\right)=0
$$

In this equation, the superscript $n+1$ is applied for time level $(n+1)$. For $\frac{d}{d t}$, by using the implicit backward difference, by considering the order of accuracy of $k$, the following equation is achieved:

$$
\frac{d}{d t} \approx \frac{1}{\Delta t} \sum_{q=1}^{k} \frac{1}{q}\left[\Delta^{-}\right]^{q}
$$

where:

$$
\Delta^{-} \mathbf{w}^{n+1}=\mathbf{w}^{n+1}-\mathbf{w}^{n} .
$$

Considering the second order accuracy, the following equation can be obtained:

$$
\frac{3 \mathbf{w}_{i}^{n+1}}{2 \Delta t_{i}}-\frac{4 \mathbf{w}_{i}^{n}}{2 \Delta t_{i}}+\frac{\mathbf{w}_{i}^{n-1}}{2 \Delta t_{i}}+\mathbf{R}_{i}\left(\mathbf{w}^{n+1}\right)=0,
$$

where $\mathbf{w}_{i}^{n+1}$ is nonlinear and, so, cannot be solved by analytical methods. To overcome this problem, a new residual, $R^{*}$, is defined and referred to the unsteady residual:

$$
\begin{aligned}
\mathbf{R}_{i}^{*}\left(\mathbf{w}^{n+1}\right)= & \frac{3 \mathbf{w}_{i}^{n+1}}{2 \Delta t_{i}}-\frac{4 \mathbf{w}_{i}^{n}}{2 \Delta t_{i}}+\frac{\mathbf{w}_{i}^{n-1}}{2 \Delta t_{i}} \\
& +\mathbf{w}_{i}^{n+1}\left(\sum_{j=1}^{m} a_{i j} \Delta x_{t, i j}+\sum_{j=1}^{m} b_{i j} \Delta y_{t, i j}\right) \\
& +\sum_{j=1}^{m} \Delta \mathbf{H}_{i j}^{I}\left(\mathbf{w}^{n+1}\right) \\
& -\frac{\operatorname{Ma}_{\infty}}{\operatorname{Re}_{\infty}}\left[\sum_{j=1}^{m} \Delta \mathbf{H}_{i j}^{V}\left(\mathbf{w}^{n+1}\right)\right] \\
& -\mathbf{D}_{i}\left(\mathbf{w}^{n+1}\right) .
\end{aligned}
$$

This equation can be used to solve steady-state problems by considering a new pseudo time $(\tau)$.

$$
\frac{\partial \mathbf{w}_{i}^{n+1}}{\partial \tau}+\mathbf{R}_{i}^{*}\left(\mathbf{w}^{n+1}\right)=0 .
$$

To solve the steady-state problem, one can have:

$$
\frac{\partial \mathbf{w}_{i}^{n+1}}{\partial \tau}=0
$$

By comparing two equations, we can have $\mathbf{R}_{i}^{*}\left(\mathbf{w}^{n+1}\right)=$ 0 , which can be used to solve Eq. (24). By considering time marching methods, such as the Runge-Kutta method [23], the solution can be found.

In this research, implicit and explicit CFL numbers are assumed to be 100000 and 5, respectively $[17,28]$. To solve Euler and Navier-Stokes equations at a solid boundary, it is assumed that the 


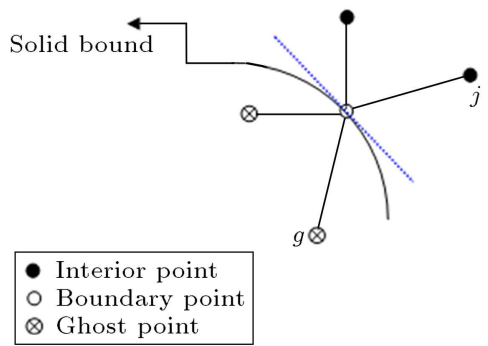

Figure 2. Schematic of boundary zone.

boundary is reflective and impenetrable [17], which can lead to the following assumption for a solid boundary:

$$
u_{n}=0, \quad \frac{\partial u_{t}}{\partial n}=0, \quad \frac{\partial H}{\partial n}=0, \quad \frac{\partial \rho}{\partial n}=0, \quad \frac{\partial P}{\partial n}=0 .
$$

To achieve a better result, especially in the solid boundary region, the Ghost point method [17] is employed. In this method, some new points are added to improve the accuracy of the mesh-less method in the solid boundary (Figure 2). For the new points, the velocity components are calculated as the following:

$$
u_{g}=-\left(u_{j}-2 \dot{x}_{b}\right), \quad v_{g}=-\left(v_{j}-2 \dot{y}_{b}\right),
$$

For viscous flow,

$$
u_{g}=u_{j}-2\left|V_{n}\right| n_{x}, \quad v_{g}=v_{j}-2\left|V_{n}\right| n_{y},
$$

For inviscid flow.

Also, in the far field, characteristic analysis based on Riemann invariants are exploited [17]. The points neighbouring stencils inside the boundary layer region and outside this area are shown in Figure 3.

\subsection{Structural model}

A NACA 0012 airfoil section model with plunging and pitching motion has been considered as a twodimensional test case of the structural model. As shown in Figure 4, the flexibility of each degree of freedom has been shown using two discretized spring models.

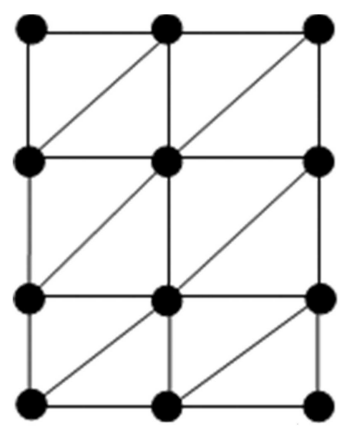

(a) In the boundary layer

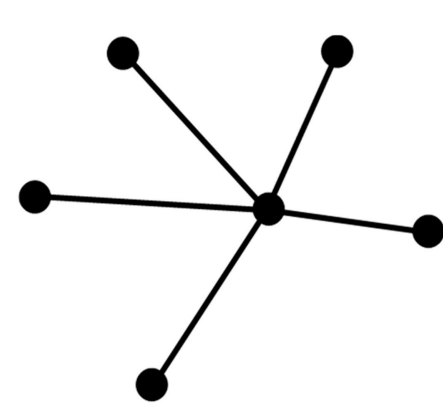

(b) In the domain outside boundary layer

Figure 3. Neighboring stencils.

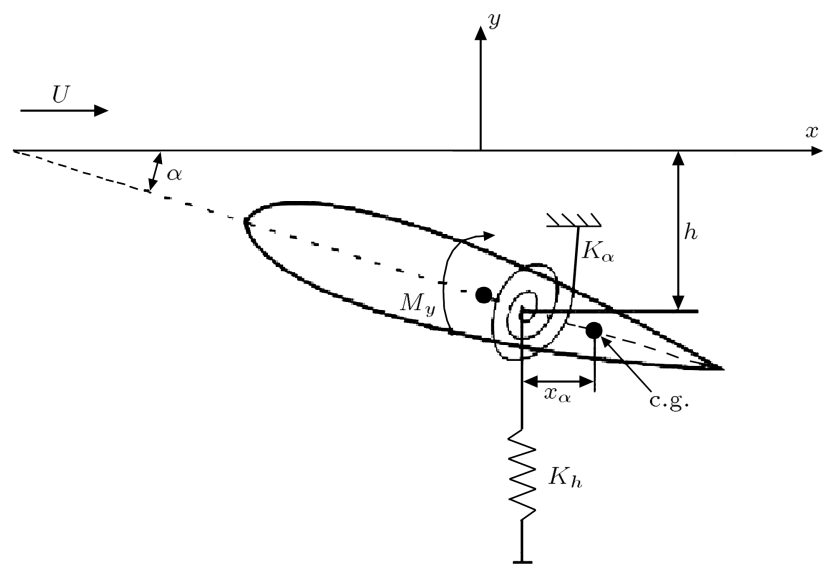

Figure 4. Typical section model.

The structural dynamics equations of motion can be written as follows [3]:

$$
\begin{aligned}
& m \ddot{h}+S_{\alpha} \ddot{\alpha}+K_{h} h=Q_{h}, \\
& S_{\alpha} \ddot{h}+I_{\alpha} \ddot{\alpha}+K_{\alpha} \alpha=Q_{\alpha} .
\end{aligned}
$$

Since the aerodynamic equations are developed in dimensionless form, it is better to use the nondimensional form of the structural equations. Thus, they can be written as [3]:

$$
\begin{aligned}
& \bar{h}+\frac{1}{2} x_{\alpha} \ddot{\alpha}+4 \frac{\omega_{r}^{2}}{\tilde{u}^{2}} \bar{h}=\frac{c_{l}}{\mu \pi}, \\
& x_{\alpha} \overline{\ddot{h}}+\frac{1}{2} r_{\alpha}^{2} \ddot{\alpha}+\frac{2 r_{\alpha}^{2}}{\tilde{u}^{2}} \alpha=\frac{4 c_{m}}{\mu \pi},
\end{aligned}
$$

where $\bar{h}$ is defined by the following relation:

$$
\bar{h}=\frac{h}{c},
$$

$\mu$ is defined as:

$$
\mu=\frac{\pi \rho b^{2}}{m} .
$$

Also, $\omega_{r}$ can be explained as:

$$
\omega_{r}=\frac{\omega_{h}}{\omega_{\alpha}}
$$

Finally, the above equations, along with the aerodynamic equations, could be incorporated into the framework of aero-elastic analysis.

\subsection{Solution methodology}

To perform aero-elastic analysis, structural and aerodynamic equations are solved sequentially. In this way, the unsteady aerodynamic loads are firstly determined using the mesh-less method for certain free-stream conditions. In the next step, these computed loads are 
applied to the structural model and they are solved by a transient dynamic analysis. Then, the obtained results, including deformations (structural dynamic response) and induced velocities, are applied to the aerodynamic model in order to update its geometry and boundary conditions for the next unsteady solution. This process continues until the user defined end condition of the problem is met. In this situation, the solution is repeated until the difference between the amplitudes of two successive picks of the alpha and displacement response become less than 0.001. For the aerodynamic solver, it is notable that at each time step, the average error should reach the level of less than 0.0001 . It must be noted that for transient dynamic analysis, the 4th order Runge-Kutta scheme is used. The flowchart of this aeroelastic solution algorithm is shown in Figure 5. Using the above mentioned methodology, an investigation is carried out about the effects of different parameters using the complete CFDstructural system.

\section{Results}

To validate the present method and show its capability for aeroelastic computations, several numerical investigations are carried out which are explained in the following subsections.

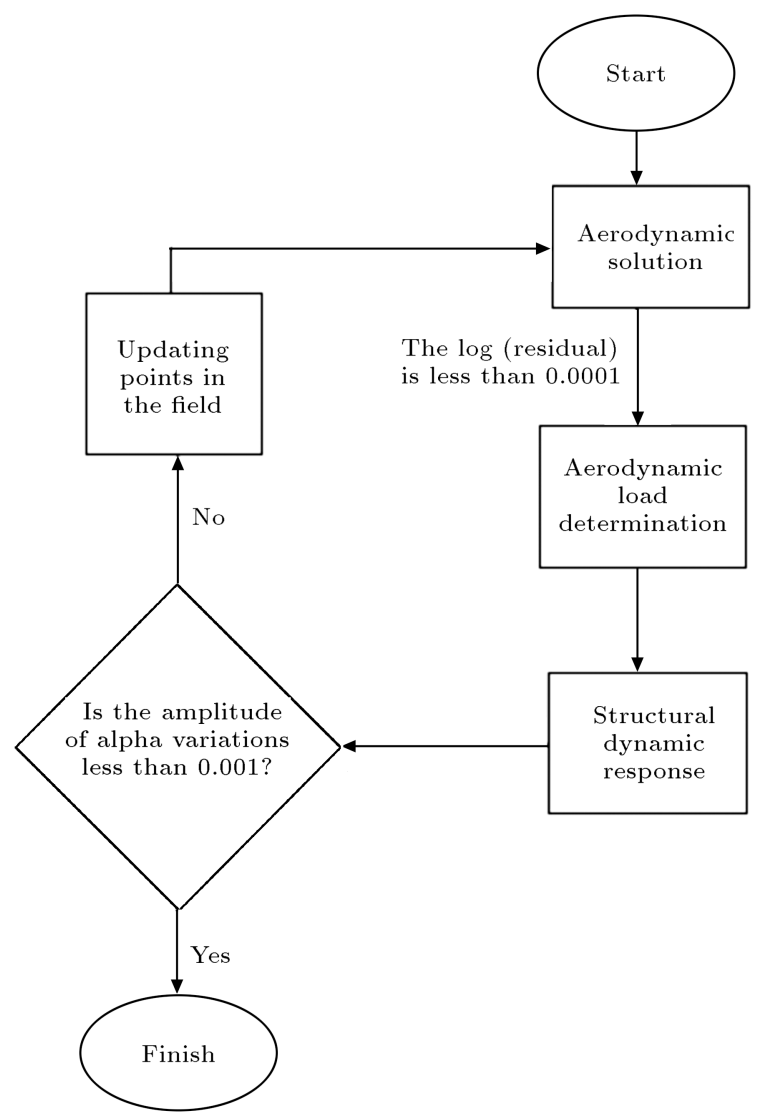

Figure 5. The flowchart of the aeroelastic analysis.

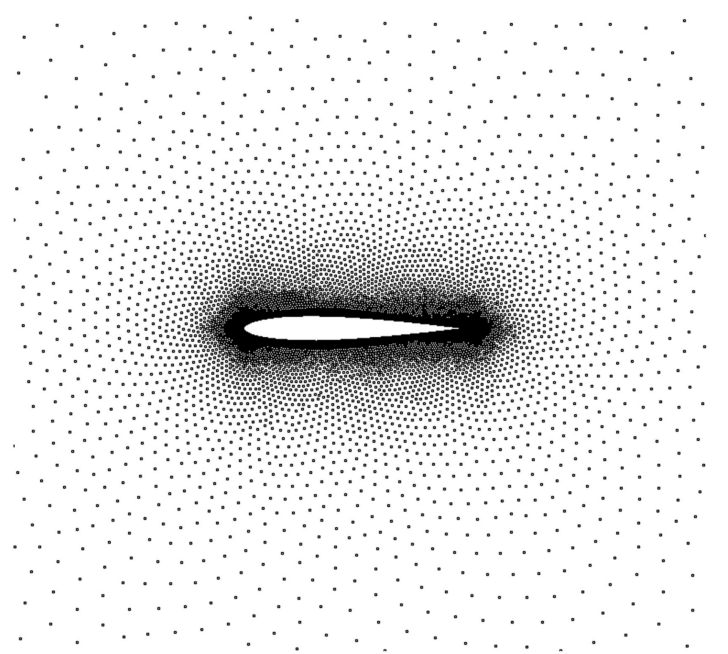

Figure 6. Point distribution around NACA0012 (viscous case).

\subsection{Viscous case}

To show the effect of viscosity and to show the ability of the method in comparison with the control volume, the first case is considered with flow conditions of $\mathrm{Ma}=0.8$ and $R e=500$. The generated point distribution around the airfoil is shown in Figure 6, which includes 13233 points in total, 72 points of which are located on the outer boundary and 364 nodes lie on the solid boundary. In this case, both Euler and Navier-Stokes solutions are obtained, and the related results are shown in Figures 7 and 8, respectively. It is notable that in this case, the amounts of dissipation terms, $\varepsilon^{(2)}$ and $\varepsilon^{(4)}$, are 0.5 and 0.015 , respectively.

In these figures, Mach number contours for different angles of attacks are shown. As illustrated, there are considerable differences between the inviscid and viscous results, which clearly show the viscosity effect in this problem. Surface pressure distributions in different situations are shown in Figure 9. As is clear, viscous terms play an important role in shock involved problems. To show the ability of the method, the convergence rate and the pressure coefficient distribution of the method at $\mathrm{Ma}=0.8$ and $\mathrm{AOA}=2.5$ are compared with similar $\mathrm{CV}$ methods with the same discretization and the same initial data [18]. Figure 10(a) illustrates that good results are achieved in comparison with the $\mathrm{CV}$ method. The convergence history is shown in Figure 10(b). As is obvious, the mesh-less method has better convergence in comparison with the $\mathrm{CV}$ method. The computations are performed on a Dual core PC with $2.00 \mathrm{GHz}$ speed. This benefit can be more helpful in aeroelastic analysis, especially in saving CPU time.

\subsection{Unsteady case}

The next case is chosen to simulate the unsteady flow solution around an oscillating NACA0012 airfoil at 


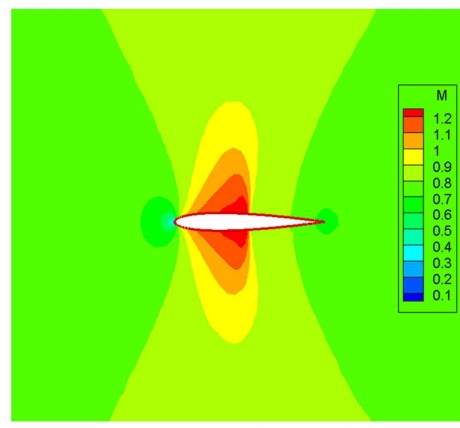

(a)

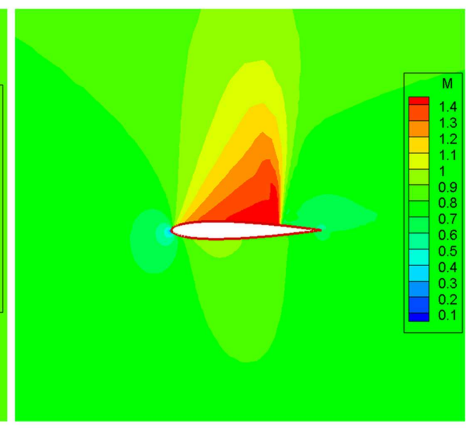

(b)

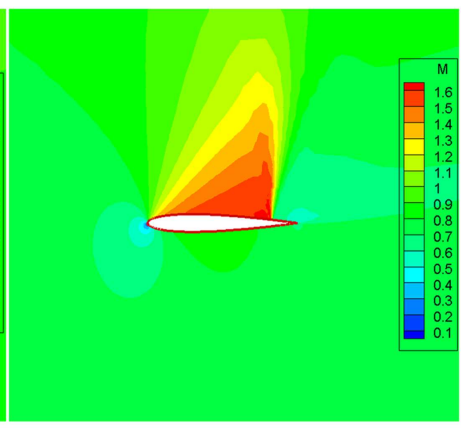

(c)

Figure 7. Mach contours around NACA0012 using Euler equations at Ma =0.8: (a) AOA=0; (b) AOA=2.5; and (c) $\mathrm{AOA}=5.0$.

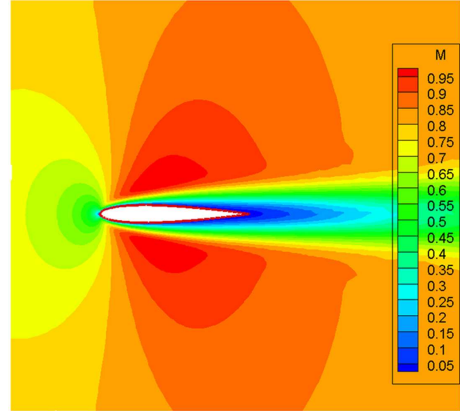

(a)

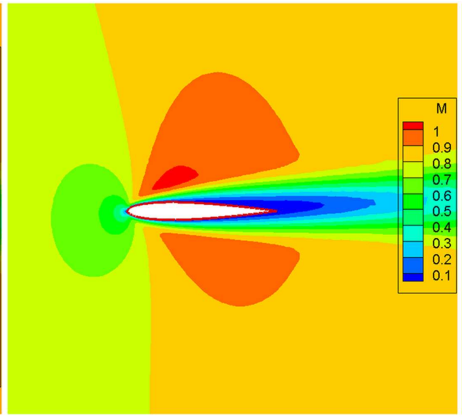

(b)

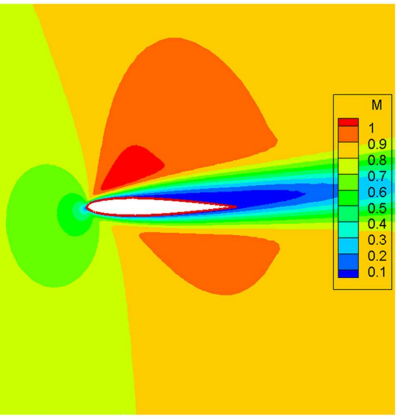

(c)

Figure 8. Mach number contours around airfoil using N-S equations at $\mathrm{Ma}=0.8$ : (a) $\mathrm{AOA}=0$; (b) $\mathrm{AOA}=2.5$; and (c) $\mathrm{AOA}=5.0$.

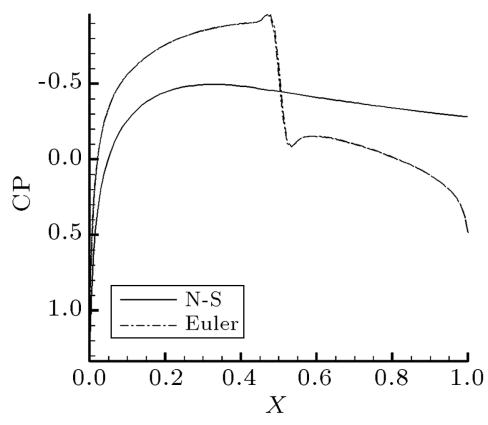

(a)

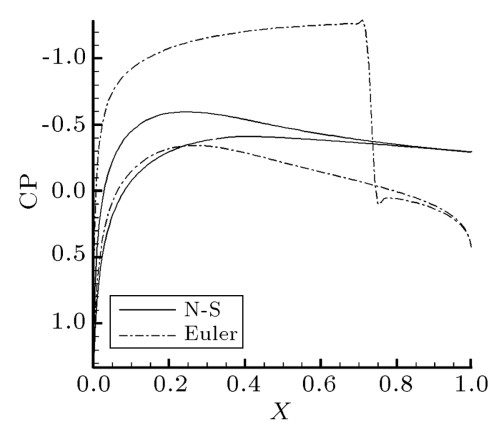

(b)

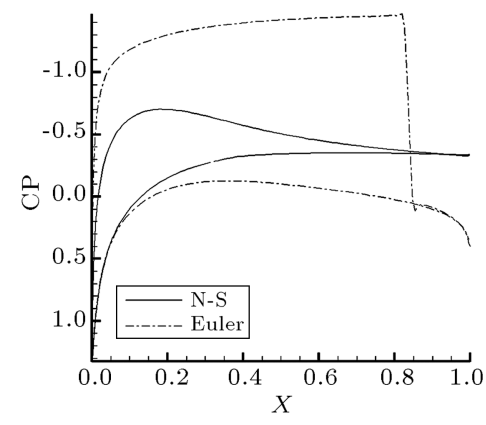

(c)

Figure 9. Surface pressure distributions at different times at $\mathrm{Ma}=0.8$ : a) $\mathrm{AOA}=0 ; \mathrm{b}) \mathrm{AOA}=2.5$; and $\mathrm{c}) \mathrm{AOA}=5.0$.

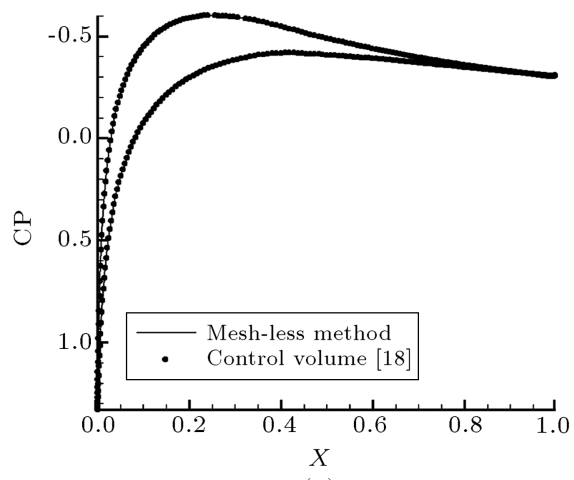

(a)

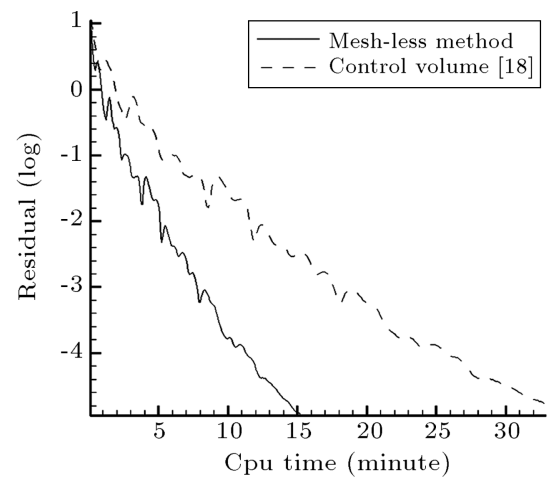

(b)

Figure 10. (a) The pressure coefficient distribution. (b) The convergence rate for NACA 0012. Ma $=0.8, \mathrm{AOA}=2.5, \mathrm{Re}$ $=500$ 


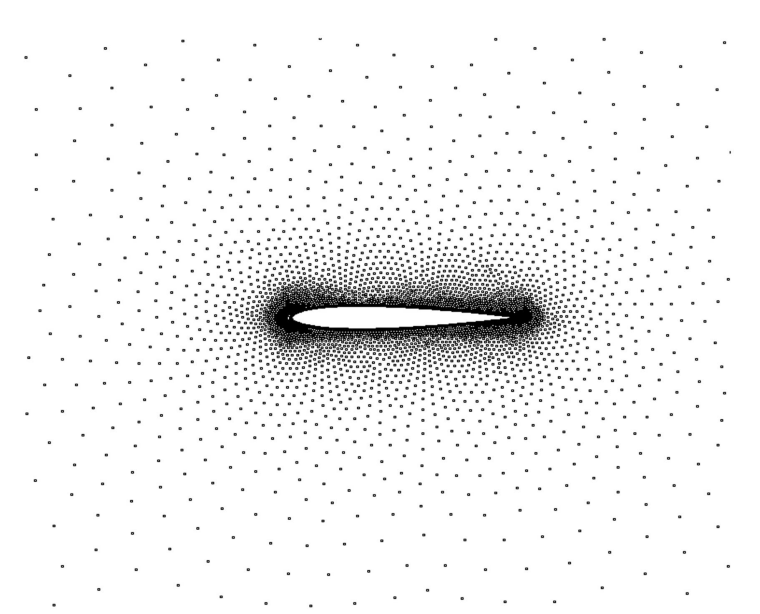

Figure 11. Point distribution model around NACA00012 airfoil.

Mach number of 0.8 . The close-view of the point distribution around the airfoil is shown in Figure 11. The point cloud contains 6509 points, of which 275 points lie on the solid boundary. The outer boundary is located 10 chords away from the airfoil with 65 points on it. The point distribution is chosen the same as in the first case. In this case, the periodic pitch angle can be considered as follows:

$$
\alpha(t)=\left(\alpha_{m}+\alpha_{0} \sin (\omega t)\right),
$$

where $\alpha_{m}$ and $\alpha_{0}$ are equal to 0.00 and 1.0 , respectively. $\omega$ can be calculated as follows:

$$
\omega=\frac{2 k U_{\infty}}{c}
$$

In this investigation, $k$ is chosen as 0.1 . Figure 12 shows normal force coefficients and pitching moment coefficients for inviscid flow. In this figure, the results are compared with CV numerical results from [18] and the experimental data of the AGARD [29]. As shown, good results are achieved in comparison with other reliable methods.
Table 1. Specifications of typical section model.

\begin{tabular}{cccc}
\hline $\boldsymbol{\rho}\left(\mathrm{kg} / \mathbf{m}^{\mathbf{3}}\right)$ & 1.2255 & $\boldsymbol{M}(\mathbf{k g})$ & 19.6245 \\
$\boldsymbol{I}_{\boldsymbol{\alpha}}\left(\mathbf{k g} \cdot \mathbf{m}^{\mathbf{2}}\right)$ & 4.1021 & $\boldsymbol{K}_{\boldsymbol{h}}(\mathbf{N} / \mathbf{m})$ & 1962.45518 \\
$\boldsymbol{K}_{\boldsymbol{\alpha}}(\mathbf{N} . \mathbf{m} / \mathbf{r a d})$ & 2563.8178 & $\boldsymbol{x}_{\boldsymbol{\alpha}}$ & 0.1 \\
$\boldsymbol{C}=\mathbf{2} \boldsymbol{b}(\mathbf{m})$ & 1.8288 & $\boldsymbol{\alpha}$ & -0.2 \\
\hline
\end{tabular}

\subsection{Flutter study}

The ability of the present method to predict aeroelastic instability is investigated by the flutter analysis of a typical section. The structural and geometrical specifications of the model are listed in Table 1.

To conduct aeroelastic analysis, firstly, the developed unsteady Euler solver is utilized for fluid computations in the coupled fluid-structure simulation. The point cloud is considered the same as in the inviscid case. Table 2 shows the obtained flutter velocity and flutter frequency of this model in comparison with other reference data [30,31]. It must be noted that the mentioned reference data are obtained using an analytical aerodynamic model (Theodorsen's theory) to capture the flutter speed. Also, for the velocity beyond this critical value, for example $v=0.3$, some snap shots of the flow field and structural response are presented in Figures 13 and 14. These figures reveal that both amplitude responses of the system increase in a rapidly progressive manner. Thus, the aeroelastic system behaves in an unstable fashion.

The other notable point is that by choosing an unsuitable time interval $(\Delta t)$, numerical instability can occur. This can affect the results and an inappropriate flutter speed, which are predicted by the present method [32]. For example, by choosing $v=$ $49 \mathrm{~m} / \mathrm{sec}$ and two different time intervals, as shown in Figure 15, different results are achieved. As is obvious, in Figure 15(a), by choosing $\Delta t=0.000001$, the amplitude response of the system increases, while in Figure 15(b), with the same initial data, and by choosing $\Delta t=0.0001$, the flutter is predicted. These

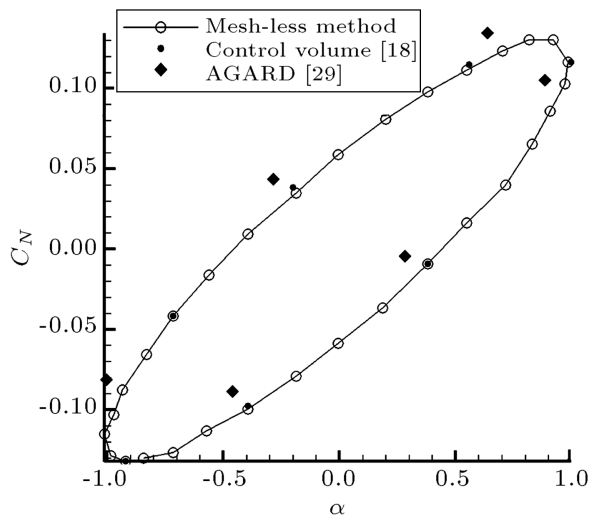

(a)

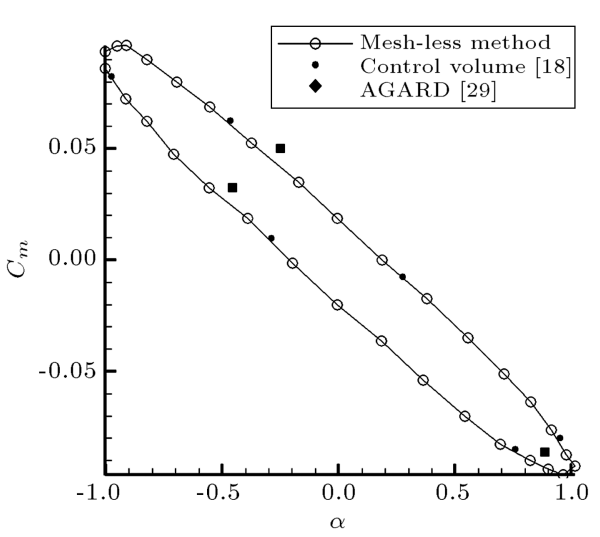

(b)

Figure 12. (a) Normal force coefficient, and b) pitching moment coefficient at Mach number of 0.8 . 


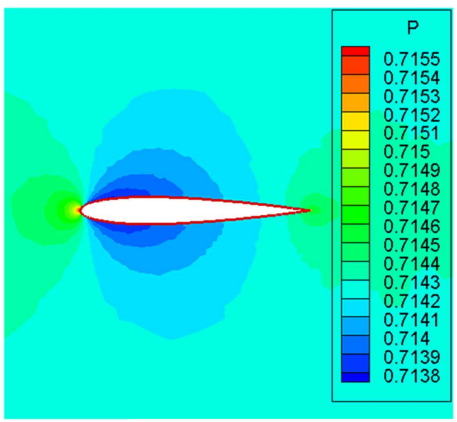

(a)

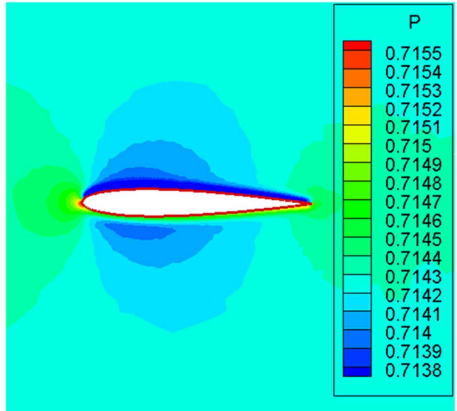

(d)

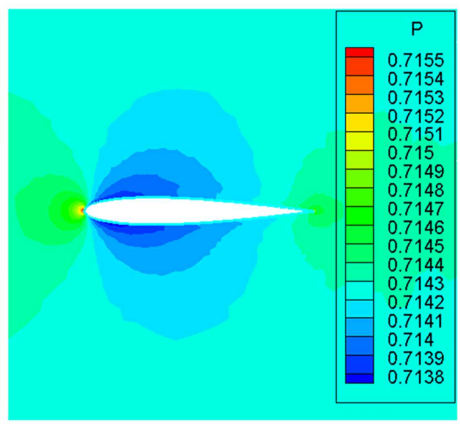

(b)

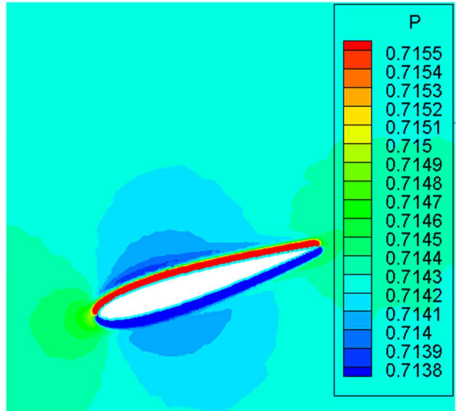

(e)

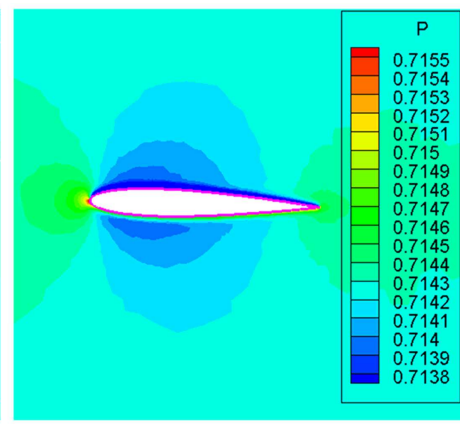

(c)

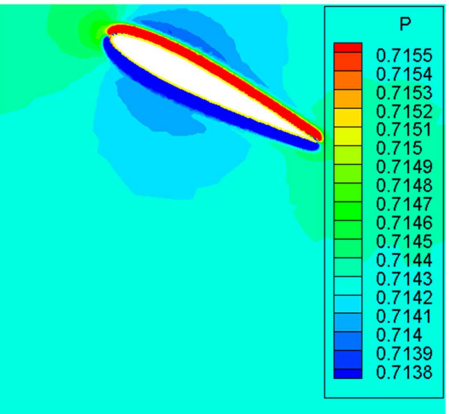

(f)

Figure 13. A few snap shots of the flow field for $v=0.3$ at (a) 1 min, (b) 2 min, (c) 3 min, (d) 4 min, (e) 4:30 min, and (f) $5 \mathrm{~min}$.

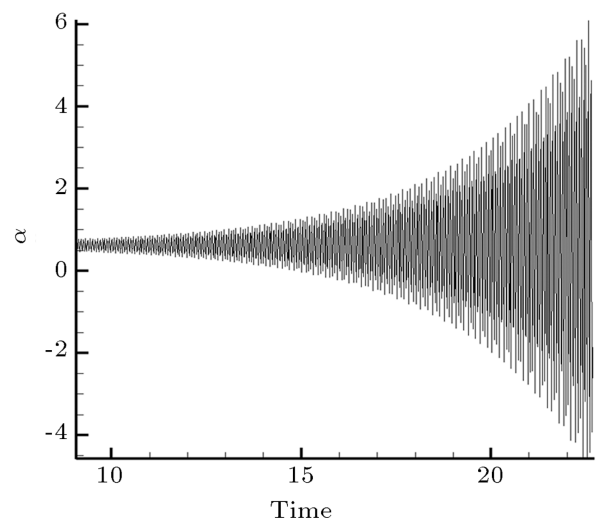

(a)

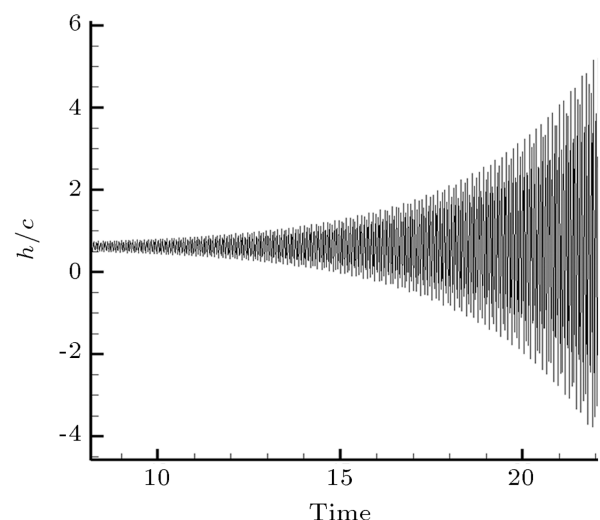

(b)

Figure 14. (a) $\alpha$ vs. time, and (b) $\bar{h}$ vs. time at $v=0.3$.

Table 2. Flutter speed of typical section model.

\begin{tabular}{cccc}
\hline & Present result & Ref. [30] & Ref. [31] \\
\hline Flutter velocity & $49.164 \mathrm{~m} / \mathrm{sec}$ & $50.5968 \mathrm{~m} / \mathrm{sec}$ & $52.42 \mathrm{~m} / \mathrm{sec}$ \\
Flutter frequency & $96.345 \mathrm{rad} / \mathrm{sec}$ & $100.19 \mathrm{rad} / \mathrm{sec}$ & $104.34 \mathrm{rad} / \mathrm{sec}$ \\
\hline
\end{tabular}

results show that a proper time interval should be chosen to prevent numerical instability.

For investigation of the ability of the present method to analyze aeroelasticity under a compressibility effect, especifically in the transonic regime, an aeroelastic analysis of the previous model, under different Mach numbers, is conducted, and the obtained results are shown in Figure 16. This figure shows that the present method, based on the Euler equation, has good agreement with Ref. [4], and confirms the acceptable accuracy of the presented mesh-less method in aeroelastic computations. It must be noted that in Ref. [4], Euler equations are applied using interpolation techniques, such as kriging and Artificial Neural Networks (ANN), to predict flutter speed in the transonic regime.

For comparing the results of different studies, the flutter index $\left(V_{F}\right)$ is defined as: 


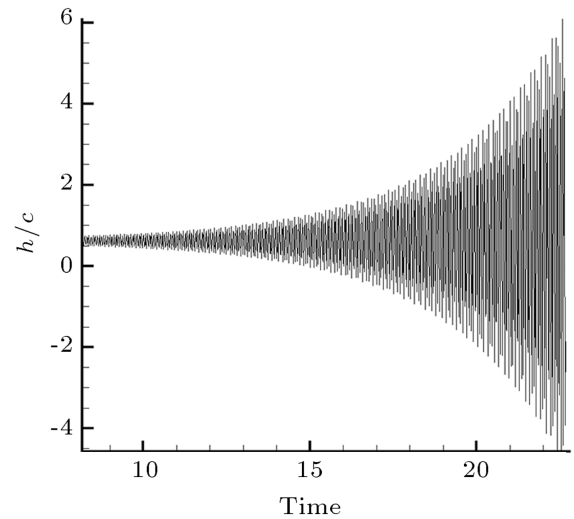

(a)

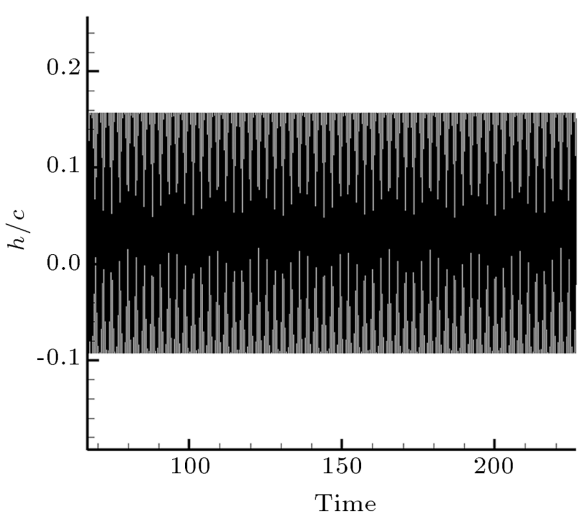

(b)

Figure 15. The effect of time interval on flutter speed predicting at (a) $\Delta t=0.000001$, and (b) $\Delta t=0.0001$.

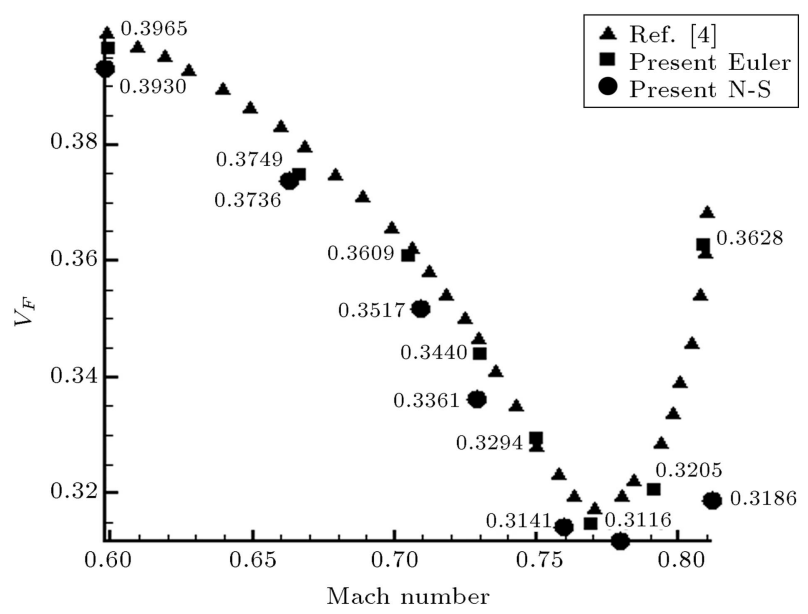

Figure 16. $V_{F}$ vs. Mach number.

$$
V_{F}=\frac{\tilde{u}}{\sqrt{\mu}}
$$

Figure 16 shows that the flutter index decreases with increasing the Mach number until critical Mach number. However, after this value, the flutter index increases sharply with increasing Mach number. The transonic dip [33] can be seen in this figure, which is because of the compressibility effects [34]. Also, the obtained results show that considering the viscosity in aeroelastic computations has little effect on the stability results if the flight Mach number is less than critical. Thus, for this flight condition, the Euler equation can be considered to overcome numerical complexity [9]. For higher Mach number, the N-S curve differences become large because, in this region, the viscous effect cannot be neglected. In this zone, the viscosity can replace the position of the shock, which has an important role to play in separation of the boundary layer. Thus, the flutter index can be affected by boundary layer separation [34]. The differences between the position of separations and shock waves in Euler and N-S equations after $\mathrm{Ma}=0.76$ creates enormous variations between the two results.

\section{Conclusions}

In this paper, a numerical aeroelastic model for a typical section (two-dimensional wing) via a mesh-less model is developed. For utilizing the time-marching technique, a dual-time implicit time discretization scheme was applied and the computational efficiency was enhanced by adopting accelerating techniques. The obtained results showed the validity of the developed solver for the fluid flow computations in comparison with available data. In addition, it was found that the time of convergence in this method is better than in the $\mathrm{CV}$ method. The ability of the method was shown by simulating the unsteady flow solution around an oscillating NACA0012 airfoil. Also, the capability of the present method to conduct aeroelastic analysis was shown using two different test cases in subsonic and compressible regimes (up to transonic regime). The results show the ability and accuracy of the present method to perform transonic aeroelastic analysis. Also, it has been shown that the viscosity effect can be neglected in transonic aeroelastic analysis when the flight Mach number is less than critical. It was shown that the choice of time interval has a significant effect on the proper prediction of flutter speed.

\section{Nomenclature}

$u \quad$ Velocity component in $x$ direction

$v \quad$ Velocity component in $y$ direction

$U \quad$ Relative velocity in $x$ direction

$V \quad$ Relative velocity in $y$ direction

$u_{n} \quad$ Normal velocity

$u_{t} \quad$ Tangential velocity

$x_{t} \quad$ Nodal velocity in $x$ direction

$y_{t} \quad$ Nodal velocity in $y$ direction

$V_{v} \quad$ Nodal velocity

$P \quad$ Pressure 
$Q_{h}$

$Q_{\alpha}$
Total energy

Distance between points $i$ and $j$

Least square coefficient in $x$ direction

Least square coefficient in $y$ direction

Artificial dissipation

Mach number in far field

Reynolds number in far field

Plunging motion

Dimensionless form of $h$

Mass of the airfoil

Chord length

Reduced frequency

Free-stream velocity

Flutter index

Static mass unbalance

Mass moment of inertia about the elastic center plunge spring constant

Pitch spring constant

Lift force

Aerodynamic pitching moment about the elastic center

Unit vector between $i$ and $j$

Static mass unbalance

Mass moment of inertia about the elastic center

Dimensionless reduced velocity

Dimensionless radius of gyration about the elastic center

A scale of distance between the center of gravity and the elastic center

Pressure sensor shock at any edges $(i j)$

Local adaptive coefficients in critical zones

Local adaptive coefficients in non-critical zones

Weighting factor

Pitching angle

Mean angle

Oscillation amplitude

Frequency of the system

Non-dimensional mass

Ratio of the specific heats

Ratio of uncoupled natural frequencies

Uncoupled plunge natural frequency

Uncoupled pitch natural frequency

Density

The average of gradients of any variable at midpoint

\section{References}

1. Garrick, I.E. and Reed, W.H. "Historical development of aircraft flutter", Journal of Aircraft, 18(11), pp. 897-912 (1981).

2. Edwards, J.W., Bennett, R.J. and Seidel, D. "Time marching transonic flutter solutions including angle of attack effects", AIAA Journal, 20(11), pp. 899-906 (1982).

3. Clark, R., Cox, D., Curtiss, H., Edwards, J., Hall, K., Peters, D., Scanlan, R., Simiu, E., Sisto, F., Strganac, T. and Dowell, E.H., A Modern Course in Aeroelasticity (Solid Mechanics and Its Applications), Springer, 4th Rev. Ed. (2004).

4. Timme, S., Rampurawala, A. and Badcock, K.J. "Applying interpolation techniques to search for transonic aeroelastic instability: ANN vs kriging", The RAES Aerodynamics Conference, Bristol, United Kindom (2010).

5. Mariem, J.B. and Hamdi, M.A. "A new boundary finite element method for fluid-structure interaction problems", Int. J. for Numerical Methods in Engineering, 24(9), pp. 1251-1267 (1987).

6. Wang, X. and Bathe, K.J. "Displacement/pressure based mixed finite element formulations for acoustic fluid-structure interaction problems", Int. J. for $\mathrm{Nu}$ merical Methods in Engineering, 40 (11), pp. 20012017 (1997).

7. Kousen, K.A. and Bendiksen, O.O. "Nonlinear aspects of the transonic aeroelastic stability problem", Presented at the AIAA/ASME/ASCE/AHS29th Structures, Structural Dynamics, and Materials Conference, Williamsburg, Virginia, DOI: 10.2514/6.19882306 (1988).

8. Lee, S. "Viscosity influence on flutter boundary and limit cycle oscillation in transonic regime", Journal of Fluid Science and Technology, 3(1) pp. 195-206 (2008).

9. Kholodar, D.B., Dowell, E.H. and Thomas, J.P. "Limit cycle oscillations of a typical section airfoil in transonic flow", Journal of Aircraft, 41(5), pp. 1067-1072 (2004).

10. Thomas, J.P., Dowell, E.H. and Hall, K.C. "Nonlinear inviscid aerodynamic effects on transonic divergence, flutter and limit cycle oscillations", AIAA Journal, 40(4), pp. 638-646 (2002).

11. Thomas, J., Dowell, E. and Hall, K. "Modeling viscous transonic limit-cycle oscillation behavior using a harmonic balance approach", Journal of Aircraft, 41(6), pp. 1266-1274 (2004).

12. Schwarz, J.B., Dowell, E.H. and Thomas, J.P. "Improved flutter boundary prediction for an isolated twodegree-of freedom airfoil", Journal of Aircraft, 46(6), pp. 2069-2076 (2009).

13. Guruswamy, G.P. "Vertical flow computations on swept flexible wings using Navier-Stokes equations", AIAA Journal, 28(12) pp. 2077-2133 (1990). 
14. Aftosmis, M.J. "Solution adaptive Cartesian grid methods for aerodynamic flows with complex geometries", von Karman Institute for Fluid Dynamics, 28th Computational Fluid Dynamics Lecture Series, 199702, Chaussée de Waterloo 72, B-1640 Rhode-SaintGenèse, Belgium (1997).

15. Liu, G.R. and Gu, Y.T., An Introduction to Mesh free Methods and Their Programming, Springer, The Netherlands (2005).

16. Katz, A. and Jameson, A. "A comparison of various meshless schemes within a unified algorithm", 47th AIAA Aerospace Sciences Meeting and Exhibit, Orlando, Florida, AIAA paper 2009-0596 (2009).

17. Jahangirian, A. and Hashemi, Y. "An efficient implicit mesh-less method for compressible flow calculations", International Journal for Numerical Methods in Flu$i d s, 67(6)$ pp. 754-770 (2011).

18. Jahangirian, A. and Hadidoolabi, M. "Unstructured moving grids for implicit calculation of unsteady compressible viscous flows", Int. J. for Num. Methods in Fluids, 47(10-11) pp. 1107-1113 (2005).

19. Wang, G., SUN, Y. and YE, Z. "Gridless solution method for two-dimensional unsteady flow", Chinese Journal of Aeronautics, 18(1), pp. 8-14 (2005).

20. Ortega, E., Oñate, E., Idelsohn, S. and Flores, R. "A meshless finite point method for three-dimensional analysis of compressible flow problems involving moving boundaries and adaptivity", Int. J. Numer. Meth. Fluids, 73(4) pp. 323-343 (2013).

21. Wang, H., Chen, H.Q. and Periaux, J. "A study of gridless method with dynamic clouds of points for solving unsteady CFD problems in aerodynamics", Int. J. Numer. Meth. Fluids, 64(1), pp. 98-118 (2010).

22. Hu, P., Kamakoti, R., Xue, L., Wang, Z. and Li, Q. "A meshless method for aeroelastic applications in ASTE-P toolset", AIA A Modeling and Simulation Technologies Conference, Toronto, Canada (2010).

23. Wendland, H. "Spatial coupling in aeroelasticity by mesh-less kernel-based methods", European Conference on Computational Fluid Dynamics ECCOMAS, (2006).

24. Sarigul-Klijn, N. "Efficient interfacing of fluid and structure for aeroelastic instability predictions", Int. J. for Num. Meth. in Eng., 47(1), pp. 705-728 (2000).

25. Lesoinne, M. and Kaila, V. "Meshless aeroelastic simulations of aircraft with large control surface deflections", AIAA paper 2005-1089, AIAA 43rd Aerospace Sciences Meeting and Exhibit, Reno, NV (2005).

26. Sattarzadeh, S. and Jahangirian, A. "3D implicit mesh-less method for compressible flow calculations", Journal of Scientia Iranica, 19(3), pp. 503-512 (2012).

27. Katz, A. and Jameson, A. "Edge-based meshless methods for compressible flow simulations", 46th AIAA Aerospace Sciences Meeting and Exhibit, Reno, Nevada, AIAA Paper 2008-699 (2008).
28. Jameson, A. "Time dependent calculations using multigrid, with applications to unsteady flows past airfoils and wings", AIA A Paper 91-1596, AIA A 10th Computational Fluid Dynamics Conference, Honolulu (1991).

29. AGARD Fluid Dynamics Panel "Compendium of Unsteady Aerodynamic Measurements", AGARD, R -702 (1982).

30. "MSC/NASTRAN verification problem manual", The Maclean-Schwendler Corporation (1988).

31. Haddadpour, H. and Firouz-Abadi, R.D. "Evaluation of quasi-steady aerodynamic modeling for flutter prediction of aircraft wings in incompressible flow", ThinWalled Structures, 44(9), pp. 931-936 (2006).

32. Benini, G.R., Belo, E.M. and Marques, F.D. "Numerical model for the simulation of fixed wings aeroelastic response", J. Braz. Soc. Mech. Sci. \& Eng., 26(2), pp. 129-136 (2004).

33. Isogai, K. "Transonic-dip mechanism of flutter of a sweptback wing", AIA A Journal, 17(7), pp. 793-795 (1979).

34. Pradeepa, T.K. and Venkatraman, K. "Shockboundary layer interaction and transonic flutter", Bulletin of the American Physical Society, 65th Annual Meeting of the APS Division of Fluid Dynamics, San Diego, California, 57(17), pp. 22-28 (2012).

\section{Biographies}

Samad Sattarzadeh received his MS degree in Aerospace Engineering from Amirkabir University of Technology (AUT), Tehran, Iran, where he is currently, a $\mathrm{PhD}$ degree student. His research interests include mesh-less methods.

Alireza Jahangirian received a BS degree in Mechanical Engineering from Amirkabir University of Technology (AUT), Tehran, Iran, in 1988, his MS degree in Mechanical Engineering from Sharif University of Technology, Tehran, Iran, in 1992, and a PhD degree from Manchester University, England, in 1997. He is currently Associate Professor in the Department of Aerospace Engineering at AUT. His research interests include: computational fluid dynamics, grid generation and evolutionary aerodynamic optimization.

Hossein Shahverdi received BS, MS and PhD degrees in Aerospace Engineering from Amirkabir University of Technology (AUT), Tehran, Iran, in 1997, 2000 and 2006. He is currently Assistant Professor in the Department of Aerospace Engineering at AUT. His research interests include aeroelasticity, structural dynamics and structural analysis. 